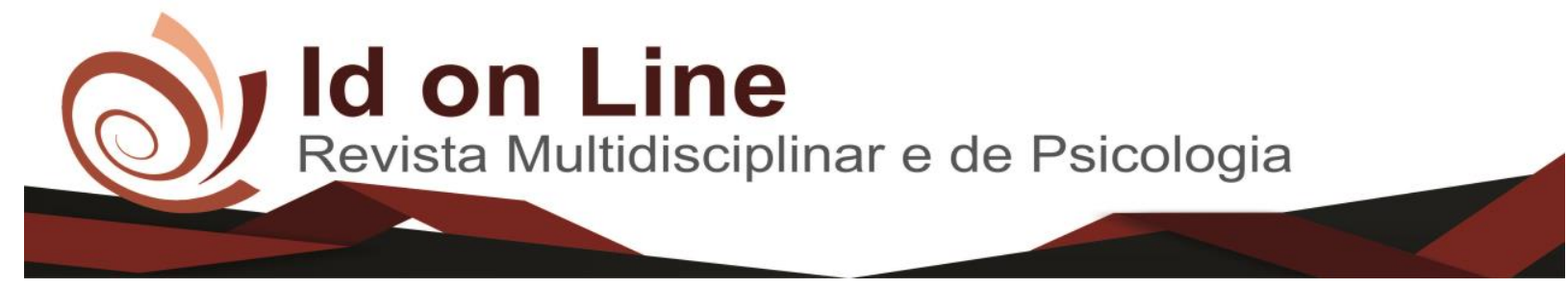

Artigo

\title{
Perfil Sociodemográfico e nível de dependência funcional de idosos com risco de quedas
}

\author{
Fabiana Ferreira Oliveira Lima ${ }^{1}$; Juliana Barros Ferreira ${ }^{2}$; Luciana Araújo dos Reis ${ }^{3}$; Kleyton Trindade Santos ${ }^{4}$; \\ Leonardo da Silva Lima ${ }^{5 ;}$ Karla Cavalcante Silva de Morais ${ }^{6}$.
}

\begin{abstract}
Resumo: O envelhecimento compreende mudanças biológicas e psicológicas que interferem na adequação ao espaço gerando risco de quedas e resultando em maior dependência funcional. Esta pesquisa teve como objetivo geral: Avaliar as variáveis sociodemográficas e nível de dependência funcional dos idosos que apresentam risco de quedas. Utilizou-se metodologia descritiva, exploratória e quantitativa com 50 idosos que obtiveram estado cognitivo preservado que apresentaram risco de quedas. Foram aplicados questionários sociodemográfico: Mini Exame do Estado Mental, índice de Barthel, índice de Lawton, avaliação antropométrica, escala de mobilidade e flexibilidade e escala Time up and Go. Neste estudo observou-se quanto à dependência funcional, que $68 \%$ mostraram-se capazes para realizar atividades e $32 \%$ apresentaram dependência parcial para a realização das mesmas. Os resultados desta pesquisa podem contribuir para a construção de ações de educação e políticas de saúde na prevenção de quedas na população idosa.
\end{abstract}

Palavras-chave: Envelhecimento. Quedas. Saúde Pública.

\section{Sociodemographic profile and level of functional dependence of the elderly at risk of falls}

\begin{abstract}
Aging involves biological and psychological changes that interfere in the adequacy to the space, generating risk of falls and resulting in greater functional dependence. This research had as general objective: To evaluate the sociodemographic variables and level of functional dependence of the elderly who are at risk of falls. Descriptive, exploratory and quantitative methodology was used with 50 elderly individuals who obtained a preserved cognitive state that presented risk of falls. Socio-demographic questionnaires were applied: Mini Mental State Examination, Barthel Index, Lawton Index, anthropometric assessment, mobility and flexibility scale, and Time Up and Go scale. In this study, functional dependence was observed, $68 \%$ to perform activities and $32 \%$ had partial dependence to perform them. The results of this research can contribute to the construction of education actions and health policies in the prevention of falls in the elderly population.
\end{abstract}

Keywords: Aging. Falls. Public health.

\footnotetext{
${ }^{1}$ Discente de Fisioterapia da Faculdade Independente do Nordeste - FAINOR. E-mail:anefaby@ hotmail.com Vitória da Conquista, Bahia, Brasil.

${ }^{2}$ Fisioterapeuta. Mestranda em Tecnologias em Saúde pela EBMSP/BA. Docente da Faculdade Independente do Nordeste - FAINOR e Faculdade de Tecnologia e Ciências - FTC Campus Vitória da Conquista- BA. E-mail: julibarros78@hotmail.com Vitória da Conquista, Bahia, Brasil.

${ }^{3}$ Fisioterapeuta. Doutora em Ciências da Saúde pela UFRN. Docente da Universidade Estadual do Sudoeste da Bahia - UESB e da Faculdade Independente do Nordeste - FAINOR. E-mail: lucianareisfainor@ gmail.com Vitória da Conquista, Bahia, Brasil.

4 Fisioterapeuta. Mestre em Ciências da Saúde pela UESB. Docente da Faculdade Independente do Nordeste - FAINOR. E-mail: kleyton_santos@hotmail.com Vitória da Conquista, Bahia, Brasil.

${ }_{5}^{5}$ Discente do curso de Fisioterapia da Faculdade Independente do Nordeste-FAINOR/BA. leooliima4@ gmail.com;

${ }^{6}$ Fisioterapeuta. Mestre em Saúde Pública pela ENSP/FIOCRUZ. Docente da Faculdade Independente do Nordeste - FAINOR e Maurício de Nassau Campus Vitória da Conquista-BA. E-mail: karlinhakau@ hotmail.com Vitória da Conquista, Bahia, Brasil.

Autor correspondente: Fabiana Ferreira Oliveira Lima. Avenida do Contorno nº 145 casa 129, Condomínio Parque Vitória - Vitória da Conquista-BA; CEP: 45.012.560 anefaby@ hotmail.com. Tel: (77) 98811-3603.
} 


\section{Introdução}

O envelhecimento da sociedade é hoje uma realidade mundial existente nos países avançados e que vem crescendo cada vez mais em países subdesenvolvidos. Isto ocorre pelo fato da transferência epidemiológica, da diminuição dos números de fecundidade, e também dos de mortalidade, levando a um aumento do número de indivíduos idosos (FERREIRA et al., 2012). O crescente número de idosos no Brasil, resulta na mudança do perfil das necessidades sanitárias, uma vez que torna-se mais frequentes as doenças crônicas degenerativas, transtornos mentais, doenças cardíacas, câncer e estresse na velhice. Essas doenças na maioria das vezes necessitam de um tratamento medicamentoso, que pode provocar como consequências efeitos adversos como instabilidade e desequilíbrio, fatores que vão favorecer a queda (REZENDE et al., 2012).

Gasparotto et al. (2014), diz que o processo de envelhecer trás ao corpo humano alterações importantes. No idoso é natural que ocorra diminuição da massa muscular que consequentemente reduz a força, conjuntamente a isso, ocorre à diminuição da massa óssea, o que gera o enfraquecimento do componente esquelético do indivíduo deixando-o frágil. Esses fatores podem comprometer a postura, a marcha, o equilíbrio e a propriocepção, condições essas que irão favorecer a queda.

Segundo Ferreira et al. (2012), o envelhecimento é compreendido como um sistema de mudanças biológicas e psicológicas. Tais alterações se refletem na contínua diminuição da competência de adequação ao espaço a sua volta, ao aumento da idade, ao risco de quedas e o surgimento de processos patológicos, que podem levar à fragilidade, e deixar o indivíduo suscetível a um ciclo vicioso patológico, que se não for interrompido pode levar o idoso a óbito.

A queda é determinada como um dos grandes problemas de Saúde Pública, sendo descrita como um episódio não proposital que tem como resposta a alteração do inicio do posicionamento de uma pessoa para um mesmo plano ou plano mais baixo, resultantes de causas multifatoriais, levando a uma incapacidade funcional gerando consequências graves no envelhecimento (GASPAROTTO et al., 2014).

Ferreira et al. (2012) explicam que a capacidade funcional pode ser definida como a manutenção da autonomia em realizar Atividades Básicas da Vida Diária (ABVD) e Atividades Instrumentais da Vida Diária (AIVD), necessárias e suficientes para uma vida 
independente e autônoma, pois para o idoso, a realização das ABVD aparece como algo presente e necessário para a sua sobrevivência, mantendo sua participação na gestão e nos cuidados com a própria saúde, e no desenvolvimento de tarefas domésticas.

A ocorrência da fragilidade em idosos está ligada ao baixo nível socioeconômico e ao baixo nível escolar. Destacam-se as causas sociais como: baixa renda, pouco estudo, a falta de amparo social e os hábitos de vida agravam a fisiopatologia da vulnerabilidade, deixando os idosos suscetíveis a cair. Contudo, é importante dizer que intervenções adequadas dessas causas são possíveis reverter o quadro de vulnerabilidade (DUARTE, 2013).

Os elevados números de incidências de quedas geram como consequência um alto custo para o sistema de saúde, evidenciando a necessidade de promover medidas de prevenção com a intenção de proporcionar soluções a nível social e individual decorrentes das quedas. $\mathrm{O}$ Sistema Único de Saúde (SUS) tem registrado a cada ano mais de 51 milhões com os custos para o tratamento de fraturas decorrentes de quedas (RODRIGUES et al., 2015).

Diante do exposto, este estudo torna-se relevante no ordenamento social, por se tratar de um assunto de interesse público envolvendo saúde e sociedade. Com os resultados desta pesquisa torna-se possível o repasse de informações para a equipe da UBS, a fim de aprimorar o planejamento dos serviços de saúde direcionados para as principais dificuldades relatadas pelos idosos, podendo colaborar na construção de políticas e ações de educação em saúde para o público idoso. Esta pesquisa teve como objetivo geral: Avaliar as variáveis sociodemográficas e nível de dependência funcional dos idosos que apresentam risco de quedas e como objetivos específicos, verificar a prevalência do risco de quedas em idosos e mensurar através de escalas validadas o nível de dependência funcional dos idosos.

\section{Metodologia}

A pesquisa foi do tipo descritiva e exploratória, com abordagem quantitativa. Os dados foram coletados em uma Unidade Básica de Saúde, situada no município de Vitória da Conquista na região do Sudoeste da Bahia. A população do estudo foi representada por 120 idosos cadastrados na Unidade Básica de Saúde. Contudo, a amostra do estudo foi representada por 50 idosos cadastrados que se enquadraram nos critérios de inclusão da pesquisa. Como critério de inclusão: idosos que apresentavam risco de quedas e que 
obtiveram estado cognitivo preservado, avaliados através do exame mini-exame da saúde mental (MEEM) e que apresentaram risco de quedas (FOLSTEIN, FOLSTEIN e McHUGR, 1979). Foram excluídos do estudo aqueles idosos que se mostraram incapazes de compreender as perguntas, mesmo apresentando um estado cognitivo adequado de acordo com o MEEM.

Foi utilizado como instrumento de pesquisa um questionário abrangente composto por variáveis sociodemográficas, bem como avaliações através da aplicação de escalas validadas: Índice de Barthel, Índice de Lawton, Mini Exame do Estado Mental, Avaliação antropométrica, Escala de Mobilidade e Flexibilidade e Escala Timed up and go (TUG). Os resultados obtidos foram expostos através de tabelas, utilizando o programa estatístico Statistical Package for Social Sciences (SPSS), versão 21.0, bem como textos descritivos.

O índice de Barthel é um instrumento de avaliação das atividades da vida diária (AVDs) e mede independência funcional no cuidado pessoal e mobilidade. E na reabilitação, tem sido usado para presumir o tempo de hospitalização, estabelecer prognósticos, adiantar consequências da alta e como instrumento de avaliação (FERREIRA et al., 2014).

O índice de Lawton e atividades instrumentais de vida diária, é composta por nove atividades onde o sujeito é avaliado de acordo com o seu desempenho e participação. Essas atividades incluem o uso do telefone, fazer compras, preparação dos alimentos, o trabalho doméstico, lavar roupas, a locomoção fora de casa, à responsabilidade com a medicação e autonomia nas finanças. A escala de Lawton tem sido empregada tanto em seu formato completo como em formato abreviado, sendo um instrumento dirigido à avaliação da funcionalidade do idoso em atividades cotidianas mais complexas, frente às atividades básicas (OLIVEIRA e MATTOS, 2012).

O Mini Exame do Estado Mental - MEEM foi desenvolvido nos Estados Unidos da América e publicado em 1975. Esse instrumento é usado para avaliar o estado mental e principalmente sintomas de demência. Foi criado pela a necessidade de uma avaliação padronizada, simplificada, reduzida e de rápida aplicação (MELO e BARBOSA, 2015).

A Avaliação antropométrica consiste em um conjunto de técnicas utilizadas para medir o corpo humano ou suas partes. Esta avaliação é de extrema importância para a identificação das alterações que acompanham o envelhecimento e se refletem no estado nutricional e no possível desenvolvimento de doenças (BUENO et al., 2008). 
A escala de Mobilidade e Flexibilidade avalia os déficits no desempenho motor, que de acordo com Ruzene e Navega (2014) se devem às alterações fisiológicas ocasionadas pelo envelhecimento.

A escala TUG (Timed up and go), permite não apenas avaliar o risco de queda de um paciente, como também a capacidade de transferência, relacionando com o equilíbrio dinâmico. Consiste em levantar-se de uma cadeira, sem ajuda dos braços, andar a uma distância de três metros, dar a volta e retornar (BARRY et al., 2014).

Foi realizada análise descritiva das variáveis de estudo, mediante a distribuição de frequência absoluta e relativa para as variáveis categóricas e medidas de tendência central (média, mediana) e dispersão (desvio-padrão), para as variáveis contínuas. As diferenças estatísticas entre proporções foram avaliadas usando-se os testes de qui-quadrado de Pearson, com um nível de significância de 5\% (p<0,05).

Para as devidas análises dos dados, foram utilizados os teste estatísticos: Teste t pareado, Teste Kolmogorov-Smirnov, Teste t de Studant e Teste de Mann-Whitney.

O estudo faz parte de um projeto mãe, cujo título: Quedas e Fatores Associados em Idosos, foi aprovado pelo Comitê de Ética em pesquisa (CAAE: 62170516.40000.5578), conforme rege a resolução 466/12. Esta pesquisa respeitou os princípios estabelecidos pela Resolução 466/12 no tocante à autonomia, beneficência, não maleficência, justiça e equidade. Tendo o compromisso de zelar pela privacidade e pelo sigilo das informações obtidas e utilizadas para o desenvolvimento da pesquisa. Todos os idosos foram esclarecidos acerca do objetivo do projeto e somente participaram aqueles que assinaram o Termo de Consentimento Livre Esclarecido.

\section{Resultados}

A amostra participante do estudo foi representada por 50 idosos cadastrados na Unidade Básica de Saúde, incluindo os idosos que apresentavam risco de quedas e que obtiveram estado cognitivo preservado e excluindo aqueles idosos que se mostraram incapazes de compreender as perguntas, mesmo apresentando um estado cognitivo adequado. 
Tabela 1: Frequência e análises sociodemográficas - geral. Vitória da Conquista/BA, 2017.

\begin{tabular}{|c|c|c|c|}
\hline Variável & Classe & $\mathrm{N}$ & $(\%)$ \\
\hline \multirow[t]{2}{*}{ Idade } & $<70$ anos & 29 & $(58,00)$ \\
\hline & $>=70$ anos & 21 & $(42,00)$ \\
\hline \multirow[t]{2}{*}{ Sexo } & Masculino & 17 & $(34,00)$ \\
\hline & Feminino & 33 & $(66,00)$ \\
\hline \multirow[t]{4}{*}{ Estado Civil } & Solteiro & 10 & $(20,00)$ \\
\hline & Casado & 24 & $(48,00)$ \\
\hline & Divorciado & 3 & $(6,00)$ \\
\hline & Viúvo & 13 & $(26,00)$ \\
\hline \multirow[t]{3}{*}{ Raça } & Branco & 29 & $(58,00)$ \\
\hline & Pardo & 18 & $(36,00)$ \\
\hline & Negro & 3 & $(6,00)$ \\
\hline \multirow[t]{10}{*}{ Profissão } & Aposentado & 14 & $(28,00)$ \\
\hline & Dona de casa & 15 & $(30,00)$ \\
\hline & Professor & 2 & $(4,00)$ \\
\hline & Missionário & 1 & $(2,00)$ \\
\hline & Lavrador & 8 & $(16,00)$ \\
\hline & Doméstica & 3 & $(6,00)$ \\
\hline & $\begin{array}{l}\text { Agente } \\
\text { comunitário }\end{array}$ & 2 & $(4,00)$ \\
\hline & Carpinteiro & 2 & $(4,00)$ \\
\hline & $\begin{array}{l}\text { Auxiliar de } \\
\text { enfermagem }\end{array}$ & 2 & $(4,00)$ \\
\hline & $\begin{array}{l}\text { Representante } \\
\text { comercial }\end{array}$ & 1 & $(2,00)$ \\
\hline \multirow[t]{5}{*}{ Escolaridade } & Analfabeto & 14 & $(28,00)$ \\
\hline & Fundamental & 19 & $(38,00)$ \\
\hline & $\begin{array}{l}\text { Ens. Méd. } \\
\text { Incompleto }\end{array}$ & 4 & $(8,00)$ \\
\hline & $\begin{array}{l}\text { Ens. Méd. } \\
\text { Completo }\end{array}$ & 9 & $(18,00)$ \\
\hline & Superior & 4 & $(8,00)$ \\
\hline
\end{tabular}

Fonte: Dados da pesquisa.

No que concerne ao perfil sociodemográfico da amostra, apresentado na Tabela 1, houve uma predominância de idosos com idade menor de 70 anos (58\%), era do sexo feminino (66\%), raça branca (58\%). Quanto ao estado civil, $48 \%$ da amostra era casada e $6 \%$ divorciada. Quanto à escolaridade, verificou-se que $28 \%$ dos idosos não eram alfabetizados, 
$38 \%$ tinham ensino fundamental, $8 \%$ tinham ensino médio incompleto, $18 \%$ com ensino médio completo e $8 \%$ com ensino superior completo ou incompleto.

A apresentação dos escores para escala MEEM, em que 38,78\% dos idosos mostraram-se incapazes no que se refere ao estado mental, enquanto 61,22\% mostraram-se capazes. Na avaliação da capacidade funcional por meio da aplicação da escala de Lawton, $32 \%$ dos idosos apresentaram grau parcial de dependência, enquanto 68\% apresentaram independência. Nenhum idoso da amostra estudada apresentou grau de dependência total. O índice de massa corporal (IMC) foi calculado com base no peso e altura. Ainda sobre o IMC, $54,55 \%$ dos idosos entrevistados estão acima do peso, apenas um indivíduo tem IMC menor do que 18,5 , que caracteriza um paciente abaixo do peso ideal.

Tabela 2: Comparação de médias para os dados antropométricos e IMC. Vitória da Conquista/BA, 2017.

\begin{tabular}{lccr}
\hline & \multicolumn{3}{c}{ Idade } \\
\cline { 2 - 3 } \multicolumn{1}{c}{ Variável } & \multicolumn{1}{c}{ Média } & \multicolumn{1}{c}{$>$ Média } & P-valor \\
\hline Altura (m) & 1,59 & 1,60 & 0,83 \\
Circunferencia_braco (cm) & 26,05 & 28,80 & 0,17 \\
Circunferencia_Pant (cm) & 31,68 & 32,93 & 0,54 \\
Cintura (cm) & 84,09 & 86,83 & 0,64 \\
Peso (Kg) & 67,40 & 61,64 & 0,10 \\
\hline IMC $\left(\mathrm{kg} / \mathrm{m}^{2}\right)$ & 26,75 & 23,92 & 0,03 \\
\hline
\end{tabular}

Fonte: Dados da pesquisa.

Observa-se que, em média, o IMC é maior para os pacientes com idade inferior a 70 anos. O p-valor foi menor do que 0,05 , o que indica médias estatisticamente diferentes. Além disso, a média do IMC para os pacientes com idade igual ou mais que 70 anos foi 23,92 $\mathrm{kg} / \mathrm{m}^{2}$.

Nos demais testes de comparação de médias, quanto à idade, foi utilizado o teste de Mann-Whitney. Foi verificado, conforme a tabela 3, que os pacientes com idade inferior a 70 anos, apresentaram uma média da escala MEEM superior aos com idade igual ou maior a 70 anos. Além disso, apresentaram tempo menor nos testes sentar levantar1, sentar levantar 2 e 
pagar lápis. Observar-se também um p-valor significativo para o teste do dinamômetro, mostrando que os idosos com idade menor que 70 anos, aplicaram mais força. Quanto à escala TUG, os idosos com idade menor que 70 anos apresentaram média menor $(9,54)$ em relação aos de idade maior que 70 anos.

Tabela 3: Comparação de médias para as escalas MEEM, TUG, atividades Sentar_levantar, Pegar_Lapis e Dinamômetro. Vitória da Conquista/BA, 2017.

\begin{tabular}{lrrr}
\hline & \multicolumn{3}{c}{ Idade } \\
\cline { 2 - 4 } \multicolumn{1}{c}{ Variável } & \multicolumn{1}{c}{$>$} & \\
\hline MEEM & Média & Média & P-valor \\
TUG & 24,29 & 23,33 & 0,43 \\
Sentar_Levantar_1(s) & 9,54 & 11,41 & 0,20 \\
Sentar_Levantar_2(s) & 13,99 & 15,6 & 0,42 \\
Pegar_Lapis(s) & 13,24 & 14,69 & 0,96 \\
\hline Dinamometro & 2,90 & 4,20 & 0,11 \\
\hline
\end{tabular}

Fonte: Dados da pesquisa.

Tabela 4: Comparação de médias para as atividades da escala BARTHEL. Vitória da Conquista/BA, 2017.

\begin{tabular}{lrrr}
\hline & \multicolumn{2}{c}{ Idade } & \\
\cline { 2 - 3 } \multicolumn{1}{c}{ Variável } & \multicolumn{1}{c}{ Média } & Média & P-valor \\
\cline { 2 - 3 } \multicolumn{1}{c}{} & 10,00 & 9,76 & 0,24 \\
\hline BARTHEL_ALIMENTAÇÃO & 5,00 & 5,00 & 1,00 \\
BARTHEL_BANHO & 5,00 & 5,00 & 1,00 \\
BARTHEL_ATIVIDADE & 9,66 & 10,00 & 0,22 \\
BARTHEL_VESTIR & 10,00 & 9,29 & 0,09 \\
BARTHEL_INTESTINO & 9,83 & 8,81 & 0,03 \\
BARTHEL_URINARIO & 10,00 & 10,00 & 1,00 \\
BATHEL_TOILLET & 14,66 & 14,52 & 0,74 \\
BARTHEL_TRANSFERENCIA & 14,83 & 14,76 & 0,82 \\
BARTHEL_MOBILIDADE & 10,00 & 9,05 & 0,02 \\
BARTHEL_ESCADAS & 98,97 & 96,19 & 0,09 \\
\hline BARTHEL_TOTAL & & & \\
\hline FOn & & & \\
\hline
\end{tabular}

Fonte: Dados da pesquisa. 
Sobre a escala Barthel, como mostra a tabela 4, as médias encontradas demonstram uma grande dependência e incapacidade funcional dos idosos estudados. Foi verificado escores menores para indivíduos de maior idade, na avaliação do sistema urinário e no uso de escadas. Nas demais variáveis, não houve diferença estatística entre as médias segundo a idade.

Tabela 5: Comparação de médias para as atividades da escala LAWNTON. Vitória da Conquista/BA, 2017.

\begin{tabular}{|c|c|c|c|}
\hline & Idade & & \\
\hline & $<70$ & $>=70$ & \\
\hline Variável & Média & Média & P-valor \\
\hline LAWNTON_1 & 2,93 & 2,81 & 0,38 \\
\hline LAWNTON_2 & 2,97 & 2,81 & 0,07 \\
\hline LAWNTON_3 & 3,00 & 2,71 & 0,02 \\
\hline LAWNTON_4 & 3,00 & 2,76 & 0,04 \\
\hline LAWNTON_5 & 2,97 & 2,67 & 0,03 \\
\hline LAWNTON_6 & 2,97 & 2,86 & 0,36 \\
\hline LAWNTON_7 & 2,90 & 2,67 & 0,19 \\
\hline LAWNTON_8 & 2,90 & 2,95 & 0,48 \\
\hline LAWNTON_9 & 2,90 & 2,62 & 0,04 \\
\hline LAWNTON_TOTAL & 26,52 & 24,76 & 0,001 \\
\hline
\end{tabular}

Fonte: Dados da pesquisa.

Quanto à escala Lawnton, o p-valor do Lawnton total foi menor do que 0,05 , o que indica médias estatisticamente diferentes entre os indivíduos com idade menor que 70 anos e os de idade igual ou maior que 70 anos.

Na comparação das médias segundo o sexo, utilizando o teste t de Studant, não foi encontrado diferenças relevantes entre as médias do IMC ( $\mathrm{p}$ valor de 0,61 ). Os resultados das médias para os testes MEEM, TUG e flexibilidade e mobilidade, utilizando o teste de MannWhitney, também não apresentaram diferenças significativas entre o sexo feminino e masculino. Porém, as médias do dinamômetro apresentaram diferenças estatisticamente relevantes entre o sexo feminino $(25,39 \mathrm{~N})$ e o masculino $(37,62 \mathrm{~N})$, com $\mathrm{p}$ valor de 0,001 . As médias resultantes da aplicação das escalas Barthel e Lawnton não apresentaram diferenças relevantes entre os sexos. 


\section{Discussão}

No que diz respeito ao perfil sociodemográfico da amostra, constatou-se que a maioria era do sexo feminino. Outros estudos relatados na pesquisa Ferreira et al. (2012), realizados com amostras de idosos em Unidades de Saúde da Família também encontraram uma predominância do sexo feminino, revelando uma diferença da mortalidade entre os sexos, na população brasileira. A presente pesquisa encontrou um baixo nível de escolaridade na amostra, onde a maioria relatou ter apenas o nível fundamental, o que pode ser justificado pelo fato de que antigamente as dificuldades de acesso à educação eram bem maiores. A baixa escolaridade encontrada neste estudo certamente está relacionada com os baixos níveis de renda também encontrados. Apenas $8 \%$ referiram nível superior, enquanto $28 \%$ referiram ser analfabetos. A falta de acesso á educação e instrução, contribui diretamente para os níveis de desemprego, sub - emprego e trabalhos informais, gerando maior instabilidade financeira e consequentemente menor acesso aos cuidados, serviços de saúde e qualidade de vida. Pilger et al. (2013) relatam que quanto menor o grau de escolaridade, maior a prevalência de dependência moderada ou grave. Certamente, a baixa escolaridade encontrada na amostra do presente estudo influencia de forma negativa na funcionalidade e qualidade de vida destes idosos, uma vez que indivíduos sem instrução tem maior grau de dependência.

Verificou-se uma média de 4,58 filhos e média de 3,34 pessoas residindo no mesmo domicílio, além da maioria afirmar ser casado(a), o que torna-se um achado positivo, visto que, ainda conforme Ferreira et al. (2012), o fato de morar só tem sido associado ao declínio na qualidade de vida e ao agravamento da morbidade, podendo funcionar até mesmo como um indicador de risco de mortalidade.

Santana et al. (2015) relatam que com relação à raça, existe um maior predomínio da raça branca para a amostra estudada, o que corrobora com os dados do Instituto Brasileiro de Geografia e estatística (IBGE) (2012), em que ha maior predominância da raça branca $(55,0 \%)$ na população idosa brasileira.

Quanto aos escores para escala MEEM, mais de um terço dos idosos participantes do estudo, mostraram-se incapazes no que se refere ao estado mental. Segundo Domiciano et al. (2014), o nível de escolaridade tem influencia no déficit cognitivo, uma vez que as maiores perdas são observadas entre os idosos com menor grau de escolaridade ou analfabetas. Já em relação ao sexo, Para Chaves et al. (2017) a população idosa feminina tem maior expectativa 
de vida, entretanto maior predisposição a desenvolver demências, sendo a doença de Alzheimer a de maior incidência. Esse fato concorda com os resultados desta pesquisa, já que verifica-se uma baixa escolaridade, maioria do sexo feminino e percentual relevante de incapacidade do estado mental.

$\mathrm{Na}$ avaliação da capacidade funcional por meio da aplicação da escala de Lawton, um terço da amostra estudada apresentou algum grau de dependência, enquanto dois terços apresentou independência. Nenhum idoso desta amostra mostrou grau de dependência total. As médias foram estatisticamente diferentes entre os indivíduos com idade menor que 70 anos e os de idade igual ou maior que 70 anos, evidenciando uma dependência maior nestes últimos. Fhon et al. (2013), relatam que quanto mais velho o idoso maior será o comprometimento em relação à sua capacidade para realizar suas atividades rotineiras. O que pode ser explicado pela própria perda das habilidades físicas para a realização de diferentes atividades. Nesse sentido, Duarte et al. (2014) corroboram com esses resultados e afirmam que, à medida que se envelhece, o idoso fica mais vulnerável à ação progressiva do meio ambiente, evoluindo com maior dificuldade para desempenhar as tarefas diárias, o que pode contribuir para uma diminuição de capacidade funcional.

Oliveira e Matos (2012), explicam que a partir dos 80 anos de idade, costuma ocorrer um declínio fisiológico na capacidade de realização das atividades diárias e concordam que o sexo feminino, analfabetismo e nível cognitivo baixo estão relacionados à dependência funcional.

Bonardi et al. (2007) relatam que com o aumento da idade, aumenta a proporção de indivíduos que necessitam de auxílio para realização de atividades da vida diária (AVD), tais como transferir-se da cama para o sofá, vestir-se, alimentar-se ou cuidar da própria higiene.

A incapacidade na realização das atividades instrumentais e básicas de vida diária pode prejudicar a vida social do idoso, o que causa transtornos para ele e sua família que, implicando em uma maior demanda de tempo, dedicação e recursos financeiros para suprir as necessidades existentes.

No tocante ao IMC, foi encontrado uma média de $25,59 \mathrm{~kg} / \mathrm{m}^{2}$, semelhante ao encontrado em outros estudos, como o realizado por Soares et al. (2012) com 66,4\% de idosos apresentando sobrepeso e Reis et al. (2015), que obtiveram em sua maioria idosos classificados com excesso de peso a partir do IMC. 
Quanto à escala TUG, este estudo encontrou uma média de 10,29 segundos para a realização do teste. Do ponto de vista desta escala, este resultado representa um baixo risco de quedas para a amostra estudada. Os idosos com idade menor que 70 anos gastaram menos tempo no teste do que os demais. Ainda neste estudo, apenas um indivíduo mostrou-se incapaz de realizar o teste de mobilidade e flexibilidade. Para as atividades sentar_levantar_1 e sentar_levantar_2, foi encontrado média de 14,71 e 13,89 segundos respectivamente. E para atividade pegar_lapis, foi encontrado uma média de 3,41 segundos.

A avaliação da capacidade funcional pela escala Barthel encontrou uma grande dependência e incapacidade funcional dos idosos estudados. Com maior dependência nos indivíduos de maior idade, no que se refere ao sistema urinário e o uso de escadas. Isto, corrobora com os achados da pesquisa de Barbosa et al. (2014), sobre a capacidade funcional dos idosos e fatores associados à incapacidade, onde foi verificada uma maior frequência de dependência para a incontinência urinária. Tal fato acaba interferindo na auto estima, auto imagem e qualidade de vida do idoso. Conforme Silva (2016), essa deficiência também pode estar relacionada ao uso de medicamentos, principalmente os diuréticos, anti-hipertensivos e anticoagulantes.

Nos estudo de Santos et al. (2014), sobre os fatores associados à perda da capacidade funcional em idosos em município no Sul do país, a incontinência urinária e a incontinência fecal foram situações clínicas que apresentaram associação com a perda da capacidade funcional. Segundo os mesmos, grande parte da amostra estudada tinha incontinência urinária e relatam associação de incapacidade funcional com incontinência urinária.

Devem ser consideradas algumas dificuldades na execução da coleta de dados, uma vez que nem todos os idosos apresentam entendimento claro sobre as perguntas e, muitas vezes, necessitam de ajuda de algum familiar para complementar as respostas. Como limitação do estudo, não foi possível aumentar o tamanho da amostra, pois o número de longevos que atendiam aos critérios de inclusão foi pequeno, dessa forma, foi considerando apenas os resultados encontrados para a população em questão. Além disso, os dados permitiram fazer associações, mas sem inferências causais. Ademais, houve dificuldades em encontrar estudos recentes nesta perspectiva e que avaliam idosos longevos. 


\section{Considerações Finais}

Considerando as características da amostra estudada, como baixa renda, baixos níveis de escolaridade, grande parte dos idosos não exercem atividade remunerada e residem com algum familiar em domicílios multigeracionais; pode-se inferir que esse contexto contribui para um estado de vulnerabilidade social, causando consequências diretas à capacidade funcional destes idosos. As avaliações funcionais realizadas neste estudo, trazem subsídios para uma assistência ao idoso identificando as capacidades funcionais prejudicadas, bem como os fatores predisponentes, servindo assim, como parâmetro para melhor planejamento da sua inserção em programas e estratégias de prevenção e assistência à saúde. Por fim, como em toda pesquisa, o tema não foi esgotado, abrindo perspectivas para que trabalhos sucessivos busquem discutir temáticas envolvendo o envelhecimento, com o propósito de contribuir para a elaboração de políticas públicas mais direcionadas a esta população.

\section{Agradecimentos}

Agradeço a Deus, que me abençoou com saúde e sabedoria para superar as dificuldades. Ao meu esposo, Rodrigo, que de uma forma especial me deu força e coragem, me apoiando nos momentos de dificuldades, cuidando do nosso filho Heitor, nos momentos que mais precisei de ajuda. A todos os professores, colegas e amigos da faculdade, em especial: Shirley Queiroz e Keilini Lemos, obrigada por todo o apoio e incentivo. A minha orientadora Karla Cavalcante, pelo o apoio, pelas suas correções e sua dedicação, essa vitória é nossa! Aos meus pais e amigos que me deram suporte nessa jornada para que eu conseguisse chegar até aqui, o meu muito obrigada.

\section{Referências}

BARBOSA, B. R.; ALMEIDA, J. M.; BARBOSA, M. R. et al. Avaliação da capacidade funcional dos idosos e fatores associados à incapacidade. Ciência e Saúde Coletiva, Rio de Janeiro, v. 19, 2014. 
BARRY, E.; GALVIN, R.; KEOGH, C.; HORGAN, F.; FAHEY, T. Is the Timed Up and Go test a useful predictor of risk of falls in community dwelling older adults: a systematic review and metaanalysis. BioMed Cetral; 2014.

BONARDI, G.; SOUZA, V.B.A.; MORAES, J.F.D. Incapacidade funcional e idosos: um desafio para os profissionais de saúde. Scientia Medica, Porto Alegre, v. 17, 2007.

BUENO, J. M. et al. Avaliação nutricional e prevalência de doenças crônicas não transmissíveis em idosos pertencentes a um programa assistencial. Ciência e Saúde Coletiva, 2008.

CHAVES, R.N.; LIMA, P.V.; VALENÇA, T.D.C.; SANTANA, E.S.; MARINHO, M.S.; REIS, L.A. Perda cognitiva e dependência funcional em idosos longevos residentes em instituições de longa permanência. Cogitare Enferm. 2017; 22(1): 01-09.

DOMICIANO, B.R.; BRAGA, D.K.A.P.; SILVA, P.N.; VASCONCELOS, T.B.; MACENA, R.H.M. Escolaridade, idade e perdas cognitivas de idosas residentes em instituições de longa permanência. Rev Neurocienc. 2014;22(3) [acesso em 02 jul 2016]. Disponível: .

DUARTE, M.C.S. et al. Prevalência e fatores sociodemográficos associados à fragilidade em mulheres idosas. Revista Brasileira de Enfermagem, v. 66, n. 6, p. 901, 2013.

DUARTE, M.R.; SANTOS, M.L.T.; BATISTA, M.F.G.; MOURA, P. Efeitos do processo do envelhecimento na capacidade funcional dos idosos e suas formas de mensuração. EFDeportes.com, Revista Digital. [Internet] 2014;19 [acesso em 19 nov 2016].

FERREIRA, L.L et al. Capacidade funcional de idosos institucionalizados com e sem doença de Alzheimer. Rev. Bras.Geriart, v.17, n.3, 2014.

FERREIRA, O.G.L. et al. Envelhecimento ativo e sua relação com a independência funcional. Texto Contexto Enferm, v. 21, n. 3, p. 513-8, 2012.

FOLSTEIN, R. R. G.;FOLSTEIN, M.; MCHUGH, P. R. Reduced caloric intake following small bowel bypass surgery: a systematic study of possible causes. Psychological Medicine, v. 9, n. 01, 1979.

FHON, J.R.S.; FABRICIO-WEHBE, S.C.C.; VENDRUSCOLO, T.R.P.; STACKFLETH, R.; MARQUES, S.; RODRIGUES, R.A.P.; Quedas em idosos e sua relação com a capacidade funcional. Revista Brasileira de Enfermagem, vol.66 no.6 Brasília, 2013.

GASPAROTTO, L. P. R.; FALSARELLA, G.R.; COIMBRA, A.M.V. As quedas no cenário da velhice: conceitos básicos e atualidades da pesquisa em saúde. Revista Brasileira de Geriatria e Gerontologia, v. 17, n. 1, p. 201-209, 2014.

IBGE - Instituto Brasileiro de Geografia e estatística. PAS - Pesquisa Anual de Serviços, 2012. Disponível na internet via URL: http://www.ibge.gov.br/home/estatistica/ecnomia/comercioeservico/pas/pas2012.

MELO, D.M; BARBOSA, A.J.G. O uso do Mini-Exame do Estado Mental em pesquisas com idosos no Brasil: uma revisão sistemática. Ciência \& saúde coletiva, v 20, n 12, p. 3865-3867, 2015.

OLIVEIRA, P.H.; MATTOS, I.E. Prevalência e fatores associados à incapacidade funcional em idosos institucionalizados no Município de Cuiabá. Epidemiol. Serv. Saúde, Brasília, 2012. 
PAULA, A.F.M. et al. Avaliação da capacidade funcional, cognição e sintomatologia depressiva em idosos atendidos em ambulatórios de Geriatria. Rev Bras Clin Med. São Paulo, v.11, n.3, 2013.

PILGER, C.; MENON, M. U.; FREITAS, M, T. A. Capacidade funcional de idosos atendidos em unidades básicas de saúde do SUS. Revista Brasileira de Enfermagem, Brasília, v. 66, n. 6, p. 907913, 2013.

REIS, L. A.; REIS, L. A.; VASCONCELOS, T, G. Impacto das variáveis sociodemográficas e de saúde na capacidade funcional de idosos de baixa renda. Ciência, Cuidado e Saúde, Maringá, v. 14, n. 1 , p. 847-854, 2015.

REZENDE, C. de P.; CARRILO, M.R.G.G.; SEBASTIÃO, E.C de O. Queda entre idosos no Brasil e sua relação com o uso de medicamentos: revisão sistemática. Cad. Saúde Pública, V. 28. N. 12, 2012.

RODRIGUES, M. da S.; TEIXEIRA, H.P..; SANTOS, J.J. da S. Prevalência de quedas em idosos e suas consequências que geram internações hospitalares. 2015.

RUZENE, J. R. S., NAVEGA, M. T., Avaliação do equilíbrio, mobilidade e flexibilidade em idosas ativas e sedentárias. Revista Brasileira de Geriatria e Gerontologia, 2014.

SANTOS, A.M.; FRANCO S.; REIS, M.A.M. Fatores associados à perda da capacidade funcional em idosos em município no Sul do país, 2014.

SILVA, L.N.M. Dependência funcional, contexto social e interação familiar no cuidado ao idoso. Mossoró - RN, 2016.

SOARES, L. D. A.; CAMPOS, F. A. C. S.; ARAÚJO, M. G. R. et al. Análise do desempenho motor associado ao estado nutricional de idosos cadastrados no Programa Saúde da Família, no município de Vitória de Santo Antão-PE. Ciência e Saúde Coletiva, Rio de Janeiro, v. 17, n. 5, 2012.

\section{Como citar este artigo (Formato ABNT):}

LIMA, Fabiana F.O.; FERREIRA, Juliana B.; REIS, Luciana A. dos; SANTOS, Kleyton T.; LIMA, Leonardo da S.; MORAIS, Karla C.S de. Perfil Sociodemográfico e nível de dependência funcional de idosos com risco de quedas. Id on Line Revista ultidisciplinar e de Psicologia, 2017, vol.12, n.39, p.164-178. ISSN: 1981-1179.

Recebido: 20.11.2017

Aceito: 18.11.2017 